Editorial

\title{
Energy Efficiency and Urban Climate Adaption
}

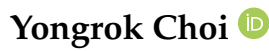

Citation: Choi, Y. Energy Efficiency and Urban Climate Adaption.

Sustainability 2021, 13, 7627.

https://doi.org/10.3390/su13147627

Received: 5 July 2021

Accepted: 7 July 2021

Published: 8 July 2021

Publisher's Note: MDPI stays neutral with regard to jurisdictional claims in published maps and institutional affiliations.

Copyright: (C) 2021 by the author. Licensee MDPI, Basel, Switzerland. This article is an open access article distributed under the terms and conditions of the Creative Commons Attribution (CC BY) license (https:// creativecommons.org/licenses/by/ $4.0 /)$.
East Asia Environment Research Center, Department of International Trade, Inha University, Incheon 22212, Korea; yrchoi@inha.ac.kr

\section{Introduction}

When the 25th Conference of the Parties to the United Nations Framework Convention on Climate Change (COP25) was held in Madrid, Spain from 2 to 13 December, 2019, there was a great expectation for the Paris Agreement to be implemented smoothly in a very transparent, predictable way. Unfortunately, this did not happen, with too many conflicts arising from the global economic depression. In particular, the withdrawal of the Trump administration of the United States caused a great deal of skepticism among all partners of the Paris Agreement. Moreover, many governments did not accomplish their commitments, and even had much more carbon emissions, even during the COVID-19 epidemic. Most policies that claimed to aim for zero-carbon economies just demonstrated superficial effects without any sustainable performance [1]. Although the weather on Earth becomes much more severe over time and almost all people of the world can now feel the aggravating effects of climate change, most developing governments and global companies have not shown proactive participation in the optimal path toward a zero-carbon economy. More seriously, especially in the short term, almost all developing countries have experienced air pollution with toxic smog. Unfortunately, all of these disasters are happening in specific areas in the world, such as metropolitan areas of developing countries. As happened 100 years ago in London, many people come to the capital cities of developing countries for a better life, but most of these rushes into the metropolitan cities have ended with too many problems resulting from the urbanization. Unfortunately, the resulting energy issues, as well as the urbanization problems, cannot be solved with traditional economic approaches because of their characteristic of market failure.

Even though there are numerous arguments about the feasibility of the Paris Agreement against climate change, the Paris regime should overcome all of the possible challenges and create a new, optimal path for all humankind, at least in terms of the global consensus on "Energy Efficiency and Urban Climate Adaptation". Many countries face urbanization issues, especially regarding the climate adaptation agenda, such as with green cities and smart cities with sustainable governance. Clearly, these issues require urgent attention from the public and private sectors in diverse fields of activity due to the complex procedures involved. There has been diverse research conducted in these fields, but most of it is too superficial, with common-sense types of empty suggestions and/or conclusions [2,3]. Now, it is time for researchers to skim the bubbles from the myths about sustainable urbanization and determine the true feasibility of the Paris Agreement for the enhancement of energy efficiency and urban adaptation. It is of the utmost importance to develop sustainable governance to solve the issues of sustainable development and sustainable management in more harmonized ways. This special issue focuses on these challenges under the Paris Agreement of 2020.

In most developing countries, government-led political regulation of energy efficiency and urban climate adaptation could be much more effective, at least in the short term and in the initial stages, but without much stronger support from the private sector, it cannot be sustainable [4]. We have already seen many policy failures at the initial stage of ecological civilization in developing countries. Therefore, this special issue seeks 
a workable mechanism for sustainable governance. Most of this paper concerns the evaluation of environmentally friendly levels of efficiency to find a workable solution against global warming, which is a major trend in environmental economics, as well as in business strategies for environmentally friendly and sustainable management [3-5]. The research question comes from efficiency- or productivity-oriented solutions based on the traditional approach toward environmental issues. This is the same for urbanization issues because there are too many problems coming from the rapidly growing metropolitan cities of developing countries, but there has only been failure to overcome these urbanization difficulties by the traditional paradigm. How can these environmental or energy issues be solved? How can developing countries overcome urbanization issues? In the following section, we shall deal with more details on the paradigm shift in the study of business and economics, and Section 3 will discuss the new research frontiers.

\section{Challenges of Energy and Urbanization Issues}

Why are the energy and environmental issues so serious? These issues cause diverse and undesirable impacts on our planet Earth, such as draught, floods, desertification, reduced life expectancy due to air pollution, etc. When environmental impacts were negligible, governments could promote their economic growth with the utmost priority, and all people in the world just strived for a successful life. This successful life could be measured in monetary terms and managed with quantitative measuring, reporting, and verification (MRV). However, there was an attack from the Earth-first with the warning of the depletion of the natural resources, and then with the devastating impact of global warming. Now, the environment is not free to share for all because the economic activities of humankind resulted in the irreparable damage of nature. Unfortunately, even with the diverse warnings from researchers and experts in the early 21st century, most governments were not concerned with the undesirable effects of energy waste and environmental aggravation. However, as the tipping point for the irreversible disaster recently came closer and closer, most governments began to realize that action is urgent and necessary. This is the background of the Paris Agreement. In recent history, it was the first consensus among all governments to mitigate the climate change in such a short time by taking much greater responsibilities for their own.

Nonetheless, there is a serious intrinsic bottleneck in the Paris Agreement, which was supported by 196 governments worldwide. Nobody wants to be the first to make too many sacrifices for energy saving and environmental protection, and most developing countries expressed that, as economic development is not optional for them, a survival kit is necessary for poor people [1]. Even though all countries agreed on the necessity of environmental protection, innovative technologies for overcoming climate change are not free. As we saw with the most recent example of the vaccine for COVID-19, even though the United States proclaimed that the medical technology for fighting against this pandemic should be freely shared among all nations, all of the private companies, as well as the governments of advanced countries, did not follow this suggestion simply because it required their contribution with a sacrifice of their potential success. Which government can persuade its people and industries to attempt this unattainable mission first? Regulation and even the free market will not solve this problem!

There have been diverse suggestions for the challenges of environmental issues, and some of those suggestions have become successful platforms for governments to enter this new world of the environmentally friendly economy. The Kyoto Protocol is the most exemplary approach to solving this public goods paradox with a market-oriented approach [1]. In this special issue, many papers have already shown the effects of this persuasive power in the energy and environmental (E\&E) research field by using this market-oriented Kyoto protocol, including its emission trading scheme (ETS) [2,5]. Unfortunately, the conclusions of their research did not match with the reality of their research subjects of governments and industries simply because most of these research papers could not find any missing links in the governance of the policies or strategies. Here, governance is defined as a work- 
able mechanism for the sustainable performance of economic agents, such as a government or industry [6]. Even though they confirmed their efforts towards E\&E issues, there was not significant improvement in any country in the world in even the most successful cases of E\&E because all of the economic agents continue to use the traditional paradigm to solve E\&E issues; thus, all of these efforts may conflict against their ultimate purpose of economic success. This is another part of the background of social responsibility.

Even though many governments, especially those of developing countries, have made great efforts towards harmonized green growth policies, they did not result in significant success in terms of energy efficiency or environmental improvement of the air because their social responsibility may be based on the traditional paradigm of minimizing inputs while maximizing outputs or profit maximization; thus, responsibility would not be voluntary, even if they said so. Many leading Korean companies have experienced terrible mishaps in terms of social responsibility. For example, there are unexpected deaths almost every day in the industrial sites due to industrial disasters. In construction sites, many people have fallen down from high places and died, and many workers have died in on the conveyor belts or under the huge pressing machines of manufacturing sites. Unfortunately, most of the people who died were not employees of the company, but rather the workers of their outsourcing companies, which were too small in size to be covered or managed by the regulatory regime. As these people simply did not account for industrial disasters, they were not covered by the insurance. Unfortunately, the original contracting companies were free from these disasters, and they were awarded as the benchmark models of corporate responsibility (CSR). Even though governments always struggle to stop these kinds of industrial disasters with strong regulations for social responsibility, there are always these kinds of missing links that allow private-sector companies to slip away. Unfortunately, these kinds of industrial disasters are the direct or indirect results of rapidly advancing urbanization, and thus, a sustainable solution to problems resulting from urbanization is desperately required as well [2].

Most developing countries, such as China, Brazil, and India, have been experiencing too many problems with urbanization. Due to the vision of the borderless competition under WTO, most developing countries have had a great opportunity to export their respective products, and this globalization of the economy has opened the new opportunity to have a richer, more successful life in metropolitan cities. Therefore, even with the strong regulations for being a resident in these cities, all developing countries have had new phenomena of urbanization. People come to metropolitan cities due to their diverse opportunities, such as the creation of new jobs and making money due to their rapidly growing economic development. Unfortunately, this rapidly advancing urbanization makes life worse for the residents of the cities by causing severe air pollution and heat dome phenomena due to the greenhouse effects of the stagnant $\mathrm{CO}_{2}$ emissions and traffic jams in cities' centers. Even though governments have made great efforts to regulate the speed of urbanization and its resulting difficulties in the cities, most policies have failed, which is typically due to the lack of governance.

Therefore, the issues of the environment, society (or social responsibility), and governance (ESG) are the new norm of the 21st century. As mentioned, most governments do provide very much financial support and public awareness for people to participate in diverse challenges with respect to the new norm of ESG, but this has resulted in heavier air pollution and skyrocketing housing prices in city centers due to the urbanization. Now, most young people cannot have hope for the next generation any longer. This is a very serious matter, and the traditional approach cannot deal with it effectively. The next section will explain all of these approaches and the paradigm shift toward the solution for this new norm of ESG. 


\section{New Frontiers of the Research on Global Green Growth}

When Adam Smith published the milestone book, The Wealth of Nations, in 1776, he created the modern principles of economics. He proclaimed that the invisible hand-the market price-could solve all economic problems more effectively than any other rules or policies. However, as the economic environment brings in much more complex and complicated issues, such as E\&E or urbanization, his market-oriented approach is no longer sufficient to solve such economic problems. These two issues of energy (or environment) and urbanization show the most exemplary failure of the traditional paradigm of economics created by Adam Smith, and thus require a paradigm shift toward a new research frontier.

Energy and its resulting environmental issues resulted in a market failure due to its character of externality as a result of climate change. A total of 197 nations in the world committed the Paris Agreement due to the worsening impact on the weather on Earth. All people in the world agreed that greenhouse gases, as well as air pollution, should be stopped, and their economies should move towards zero-carbon economies. However, unless all other people participate in this trend, they cannot feel its positive impacts, as their burden as an early adaptor is more severe than that of the late-comers. Therefore, the UNFCC and the annual meeting of the Conference of Parties (COP) created the Kyoto Protocol to overcome the environmental issues by using market-oriented approaches. By using the ETS, diverse forms of Joint Implementation, and the Clean Development Mechanism, all the partners of the COP thought that climate change could be solved at last. However, each of these did not work in its planned way, resulting in the more advanced Paris Agreement, which offered voluntary participation in the abatement of GHG emissions, rather than using a market-oriented approach. Even though this Paris Agreement still depends on various market-oriented approaches, it is noteworthy that the ultimate mission is based on the voluntary participation of all nations and all people of the world. Even though the Paris Agreement shed grand lights on human history, the problem still exists because of "voluntary participation", i.e., due to the lack of governance.

There have been numerous studies on dealing with E\&E issues, even in this special issue. However, most of these papers are based on the quantitative causal relationships between multiple inputs and outputs. For this purpose, the most popular model is that of data envelope analysis (DEA) of straight lines, which is used in Western science to evaluate the performance of a locational economy, such as a province or country, or the micro-level performance of industries or firms [4,7]. The contributions of this model are noteworthy, as it shows the great potential for improving environmentally friendly economic development and sustainable management. Nonetheless, DEA harmonizes multiple inputs and outputs for better performance based on weak disposal, but it still has the Achilles' weaknesses of articulating the weights of these causal relationships. As long as the weights are articulated subjectively, it is not appropriate to harmonize the interests of all decision-making units (DMUs). Another way to include the traditional market failure of externality in the field of E\&E comes from the computable general equilibrium (CGE) model, which uses actual economic data to estimate how an economy might react to changes in policy, technology, or other external factors [3]. However, this model also has a critical weakness in that it is based on the marginal contribution of external shock in positive terms only. It is quite easy to say that something is better than nothing, but we do not know the negative effects of the new regulations or technologies on the legacy system. Thus, the most important future direction for E\&E should come from the perspective of inclusive growth in order to include "voluntary participation" with strong governance.

Urbanization is also another extreme case of the intrinsic failure of traditional economics. The traditional economic approach is based on economies of scale; having more inputs will decrease the average cost of production, resulting in more products at a cheaper price. Thus, many factories in the in the metropolitan cities of the United Kingdom recruited many workers who lost their land due to the "Enclosure Movement" in the 19th century. During this industrialization process, the notorious London smog (smoke + fog) was an exemplary disaster that resulted from this rapidly advancing urbanization. The 
movement of more people to the cities caused heavier air pollution and created the terrible mishap of this London smog. Almost 200 years later, this happened almost everywhere in developing countries. The borderless competition under the regime of the WTO has created one global market, and most developing countries have enjoyed this golden opportunity to produce more for export; they have thus recruited many more workers not only from rural areas, but also from the other less developed countries. All of this immigration to metropolitan cities has caused historic levels of air pollution everywhere in developing countries, resulting in a faster impact on climate change, especially in metropolitan areas, due to the heat dome phenomenon. The problem has been worsened by urbanization because it is based on the principle of economies of scale. Government regulations are not easily accepted in the private sectors due to the basic motivation of economic theory. This is the reason for the private sector's hesitance and reluctance with respect to "voluntary participation" in the Paris Agreement. At the end of 2020, there were 627 million dollars of ETS debts among the top 30 companies in Korea, while their assets in ETS totaled 463 million dollars, resulting in 164 million dollars in deficits altogether because there were always fewer sellers of ETS allowances than buyers. Almost all companies want to buy emission allowances, but they do not want to sell their allowances in the ETS market because this would not be reflect in the shadow prices of carbon emissions. Samsung Electronics had 31 billion dollars in profits, but had 28 million dollars of debt for allowances in 2020. It had 11.3 million tons of free allowances but actually used 13 million tons, resulting in 1.7 million dollars of debt for allowances. This shows the intrinsic failure of policies for the regulation of environmentally friendly economic performance. In the year 2021, the allowance target was reduced from $97 \%$ to $90 \%$, causing the market to be more aggravated.

Because there has been a much stronger emphasis placed by policymakers and researchers on productivity rather than environmental performance, especially in metropolitan cities, it is much more complex to regulate the "harmonized balance" between these two aspects of economic and environmental performance simultaneously. In metropolitan cities, such as Seoul or Beijing, the traditional approach based on DEA or CGE leads to the conclusion that these cities, which are supported by urbanization, are almost on the frontier of the production possibility curve, and thus, they cannot easily regulate their air pollution issues without a significant sacrifice of their economic outputs. This is the problem of the current research on E\&E and urbanization issues-we cannot harmonize these two different missions unless we know their exact contributions to the quality of our life. The new frontier for research is inclusive growth, as well as sustainable management. Instead of the traditional maximization of the efficiency or profits for shareholders, this approach insists on the harmonization of all interests of the stake holders. Unfortunately, the traditional economic approach to E\&E and urbanization cannot provide a harmonized solution for a zero-carbon economy.

If the total output is fixed in the traditional approach, it is very difficult to harmonize all of the partners or participants in certain economic activities. Thus, the old paradigm of economics based on economies of scale or economies of efficiency (maximizing outputs while minimizing inputs) for one economic agent should be replaced with the new paradigm of value creation based on sustainable network management among stakeholders. For this new paradigm, the productivity-based solution should be replaced with an innovation-oriented solution. If everybody wants a larger pizza, we cannot divide the pizza into appropriate sizes for all. Thus, the larger pizza of innovative technology should be used in this new frontier of the research. In this special issue, several papers tried this new conceptual approach to handling environmental issues. From these papers and the basic principles of governance theory, the following suggestions can be proposed for future research.

First, the regulation of air pollution may work only in the initial stages of the paradigm shift from traditional efficiency towards inclusive growth, and only for a short time. If the partners do not agree with this new norm of inclusive growth, another balloon effect may be incurred. Thus, balanced promotion and regulation should be harmonized in the same 
direction. For example, green technology has a wide spectrum of cost-effectiveness. Some innovative green technologies, such as LED lights, could save costs when we consider all of the costs and the expected lifetimes of the lights compared with those of fluorescent lights. Nonetheless, people do not want to change their behavioral habits. Thus, regulation could be very effective for this low-cost technology. Meanwhile, carbon capture and storage technologies are not only costly, with longer times needed for their development and a much lower capacity for reducing carbon emission, but the industry will also not participate easily unless governments provide some type of matching fund as public support. Most importantly, these two regulations and promotions should go together in the same direction. Otherwise, this may give the wrong signals for moral hazards of the partners. In Korea, the government declared its strong support for renewable energy development, and at the same time, it gave some subsidies for the diesel cars to micro-businesses. These kinds of conflicting policies cannot accomplish the goal of a zero-carbon society.

Second, the leadership should show a transparent, predictable, and optimal path for all people. Leadership is the most important determinant of governance. As shown in the United States, the Trump administration made the decision to withdraw the USA from the Paris Agreement, and this became effective just a few days before his retirement. The new Biden administration cancelled this withdrawal and reentered the Paris Agreement with strong leadership. Now, not only the people of the USA, but all people of the world have a serious bottleneck in proceeding with the Paris Agreement, as there is more air pollution than ever due to the withdrawal of the United States. This kind of paradigm shift between consecutive presidents happened in Korea as well. When President Lee declared the national vision of green growth during the 2008 G20 Seoul summit, all Korean people thought that Korea may enter an era with a new norm of environmental protection. However, the next president, Park, gave passive and even pessimistic direction for green growth, and the current president, Moon, also did not show clear willingness and exhibited much confusion in his environmental policies. His administration supported renewable energy, but at the same time, it supported the construction of inland coal-fired power plants, as well as the export of such air-polluting power plants. If leadership shows these kinds of pendular policy conflicts, there will be no reliable and effective policies in the long term.

Third, a cultural revolution toward a sustainable economy is crucial. A powerful partnership between the public and private sectors should be the basic platform for the paradigm shift toward a zero-carbon society. In Korea, not all people dump out their garbage, which is due not only to the cost of plastic bags for this garbage, but also to environmental concern. If the manager of Samsung Electronics decides to receive a penalty instead of carbon abatement, then nobody in the company will have deep concern for environmental protection. Therefore, strong support for changes in habits should be based on the voluntary participation of all stakeholders of the public and private sectors. Educational training and some incentives in the initial stages will boost this new cultural revolution.

\section{Concluding Remarks}

In this special issue, most papers evaluated the performance of E\&E and urbanization policies with various and multiple inputs and outputs. Even though this paper showed the feasibility of these policies for the new norm of sustainable development and inclusive growth, there are still missing links in the enhancement of their performance due to the lack of governance. Thus, this special issue makes suggestions for three ways to promote good governance for inclusive growth. Unfortunately, none of these paths towards a zero-carbon economy can be easily quantified for the measurement, reporting, and verification of the performance. Nonetheless, these three propositions for governance will open a new frontier for future research. 
Funding: This work was supported by the National Research Foundation of Korea (Grant No. NRF-2019R1A2C1005326).

Institutional Review Board Statement: Not applicable.

Informed Consent Statement: Not applicable.

Data Availability Statement: Not applicable.

Acknowledgments: I appreciate all of the participants in the "Sustainable Asia Conference at Incheon (http:/ / abf.inha.ac.kr/, accessed on 8 July 2021)". Due to COVID-19, we could have the conference, yet all of these members shared very much for this special issue with their precious contributions.

Conflicts of Interest: The author declare no conflict of interest.

\section{References}

1. Choi, Y. Digital Business and Sustainable Development: Asian Perspectives; Rotledge Press: London, UK, 2017.

2. Lv, T.; Wang, L.; Zhang, X.; Xie, H.; Lu, H.; Li, H.; Liu, W.; Zhang, Y. Coupling coordinated development and exploring its influencing factors in Nanchang, China: From the perspectives of land urbanization and population urbanization. Land 2019, 8, 178. [CrossRef]

3. Tan, X.; Liu, Y.; Cui, J.; Su, B. Assessment of carbon leakage by channels: An approach combining CGE model and decomposition analysis. Energy Econ. 2018, 74, 535-545. [CrossRef]

4. Choi, Y.; Wang, H.; Yang, F.; Lee, H. Sustainable governance of the Korean freight transportation industry from an environmental perspective. Sustainability 2021, 13, 6429. [CrossRef]

5. Kung, C.C.; Zhang, N.; Choi, Y.; Xiong, K.; Yu, J. Effectiveness of crop residuals in ethanol and pyrolysis-based electricity production: A stochastic analysis under uncertain climate impacts. Energy Policy 2019, 125, 267-276. [CrossRef]

6. Choi, Y.; Ashurova, Z.; Lee, H. Sustainable governance on the intention of medical tourism in Uzbekistan. Sustainability 2021, 13, 6915. [CrossRef]

7. Zheng, H.; Zhang, Z.; Wei, W.; Song, M.; Dietzenbacher, E.; Guan, D. Regional determinants of China's consumption-based emissions in the economic transition. Environ. Res. Lett. 2020, 15. [CrossRef] 